\title{
Interference-Aware Transmission Scheduling for Internet of Vehicles
}

\author{
Mohammad Zubair Khan ${ }^{1, *}$, Muhammad Awais Javed ${ }^{2}$, Hamza Ghandorh ${ }^{1}$, Omar H. Alhazmi ${ }^{1}$ and \\ Khalid S. Aloufi ${ }^{3}$
}

\author{
${ }^{1}$ Department of Computer Science, Taibah University, Medina, 42353, Saudi Arabia \\ ${ }^{2}$ Department of Electrical and Computer Engineering, COMSATS University Islamabad, 45550, Pakistan \\ ${ }^{3}$ Department of Computer Engineering, College of Computer Science and Engineering, Taibah University, Medina, 42353, \\ Saudi Arabia \\ *Corresponding Author: Mohammad Zubair Khan. Email: mkhanb@taibahu.edu.sa \\ Received: 03 October 2021; Accepted: 13 November 2021
}

\begin{abstract}
Next-generation Intelligent Transportation Systems (ITS) use wirelessly connected vehicles for safety and non-safety applications such as autonomous driving, cooperative awareness, route guidance and multimedia transmissions. This network known as the Internet of Vehicles (IoVs) suffers from many challenges such as collisions due to hidden terminals, and interference from simultaneously transmitting vehicles. Moreover, the packet reception ratio of transmissions between vehicles is significantly reduced at high vehicle densities and severe fading scenarios. As safety applications require periodic broadcast of safety messages from each vehicle to all other vehicles in the neighborhood, the development of an efficient medium access technique is a key challenge. In this paper, we propose an interference-aware medium access protocol for vehicular networks that allocate time slots to the vehicles such that interference due to hidden nodes is minimized. We first provide an analytical model to compute the interference range of a vehicle in the presence of simultaneously transmitting vehicles. We also propose a scheduling algorithm that uses vehicle GPS positions and reuse the same time slot only at a distance greater than the interference range. Simulation results show that the proposed technique improves the packet reception ratio by $70 \%$ as compared to the standard IEEE $802.11 \mathrm{p}$ based CSMA/CA protocol.
\end{abstract}

Keywords: Vehicular Network; ITS; medium access control

\section{Introduction}

Wireless communication between vehicles is used to implement intelligent and smart applications of future Intelligent Transportation Systems (ITS) [1-6]. As the robustness and reliability of wireless communications have increased over the last decade, many new applications related to passenger and vehicle safety have been introduced. Future vehicles will be smart and autonomous, thus making decisions such as route planning, self-driving, and traffic management [7-9].

This work is licensed under a Creative Commons Attribution 4.0 International License, which permits unrestricted use, distribution, and reproduction in any medium, provided the original work is properly cited. 
Secure and reliable data sharing between vehicles is the essence of every ITS application. With the help of wireless technologies such as 6G and IEEE $802.11 \mathrm{p}$, data can be efficiently transmitted between vehicles with high data rates and reliability [10-15]. This is particularly useful for safety applications where vehicles periodically broadcast mobility-related data to each other and develop a map of neighboring vehicles in the neighborhood [16-20].

There are two major standards are used for vehicular communications [19]. The first is the wireless local area network-based IEEE 802.11p, and the second is the Long-Term Evolution (LTE) based Cellular Vehicle to Everything (C-V2X). IEEE 802.11p uses Carrier Sense Multiple Access with Collision Avoidance (CSMA/CA) protocol at the medium access layer. On the other hand, C-V2X uses Sensing Based Semi Persistent Scheduling (SB-SPS) protocol for managing the transmissions between vehicles in an ad hoc manner.

A major challenge to efficiently share data between vehicles is interference and collisions caused by hidden nodes. As vehicular communications for safety applications are ad hoc in nature, vehicles are not aware of simultaneously transmitting vehicles and hence can result in collisions. This is a major concern when vehicle density is high resulting in high data traffic load in the network.

In this paper, we propose an interference-aware medium access protocol that reduces the collisions caused by hidden nodes and improves the reliability of safety message transmissions. The key idea of the proposed technique is to keep the distance between simultaneously transmitting nodes greater than the interference range. Following are the major contributions of this work:

1. We developed an analytical model to evaluate the interference range of the vehicles in a hidden node scenario.

2. We proposed a novel medium access control protocol that uses interference range knowledge to schedule the safety message transmissions.

3. We evaluate the performance of the proposed technique in a realistic vehicular scenario and show the performance improvement in terms of packet reception ratio and the ratio of packets that faced collisions.

The paper is organized as follows: Section II discusses the literature review and recent work conducted in safety message transmissions. Section III describes the system model whereas Section IV explains the analytical model and the proposed technique. Section V discusses the performance evaluation and results. Section VI concludes the paper.

\section{Literature Review}

Several techniques have been proposed in the literature to improve the transmission of safety and nonsafety messages in vehicular networks. We categorize these techniques into two categories: IEEE 802.11p based and C-V2X based. In Tab. 1, we present the recent techniques in literature for both categories.

Table 1: Recent work in Literature

\begin{tabular}{llll}
\hline $\begin{array}{l}\text { Reference } \\
\text { technology }\end{array}$ & Key idea & Results \\
\hline$[1]$ & IEEE $802.11 \mathrm{p}$ & $\begin{array}{l}\text { Link-layer cooperation } \\
\text { Utilization of unused time slots }\end{array}$ & Improved packet reception ratio \\
\hline
\end{tabular}




\begin{tabular}{|c|c|c|c|}
\hline Reference & $\begin{array}{l}\text { Wireless } \\
\text { technology }\end{array}$ & Key idea & Results \\
\hline [2] & IEEE $802.11 p$ & $\begin{array}{l}\text { Multi-hop transmissions } \\
\text { Probabilistic transmissions } \\
\text { Clustering }\end{array}$ & $\begin{array}{l}\text { Reduced number of } \\
\text { transmissions }\end{array}$ \\
\hline [3] & IEEE $802.11 p$ & $\begin{array}{l}\text { Cooperative Spectrum Use } \\
\text { Transmissions based on matching theory }\end{array}$ & Improved network throughput \\
\hline [4] & $\mathrm{C}-\mathrm{V} 2 \mathrm{X}$ & $\begin{array}{l}\text { New sensing time slot } \\
\text { Sensing accuracy }\end{array}$ & Improved packet reception ratio \\
\hline [5] & $\mathrm{C}-\mathrm{V} 2 \mathrm{X}$ & $\begin{array}{l}\text { Neighborhood announcement } \\
\text { Small announcement packet } \\
\text { transmission }\end{array}$ & Reduced number of collisions \\
\hline [6] & $\mathrm{C}-\mathrm{V} 2 \mathrm{X}$ & $\begin{array}{l}\text { Adaptive transmit power selection } \\
\text { Adaptive delay between packet } \\
\text { transmissions }\end{array}$ & Reduced interference \\
\hline
\end{tabular}

In [1], the authors proposed a novel link-layer cooperative medium access control protocol. The key idea is to find relay vehicles that have unused time slots. For each transmission, relay vehicles are identified, and packets are scheduled in their free time slots. Results highlight the improved packet reception ratio achieved by the proposed technique.

The work in [2] uses multi-hop transmissions to disseminate emergency safety messages. As the communication range of the IEEE $802.11 \mathrm{p}$ is limited, hence multi-hop technique provides a higher communication range. Each relay vehicle uses probabilistic transmission to forward the message to the other nodes. In this way, the number of relay vehicles is reduced, and emergency packets are forwarded with a smaller number of transmissions.

In [3], the authors use a cooperative technique for spectrum sharing between IEEE $802.11 \mathrm{p}$ and C-V2X users. The proposed technique uses energy detection to find free slots in the spectrum domain. Furthermore, the matching theory is used to allocate free spectrum slots to vehicles. Results indicate a higher throughput achieved by the proposed technique.

The work in [4] introduces a new time slot in the C-V2X medium access protocol to better sense the simultaneous transmissions. As a result, sensing accuracy is improved and collisions due to hidden nodes are reduced. Simulation results show a higher packet reception ratio for the sensing slot-based transmissions, with a slightly increased overhead required for improved sensing.

In [5], the authors propose the use of neighborhood announcement messages before safety message transmissions. A small announcement packet is transmitted before every safety message, thus alerting other vehicles of ongoing transmission. As a result, the number of safety message collisions is reduced.

The work in [6] proposes an adaptive transmit power control scheme to reduce the interference among the safety messages. In addition, the authors also propose an adaptive delay between two consecutive safety message transmissions, thus varying the packet generation rate. The proposed technique reduces interference and improves the packet reception ratio. 
The previous work in literature use techniques such as link layer cooperation, multi-hop transmissions, cooperative use of spectrum, addition of sensing time slots, use of announcement messages, and transmit power control to mitigate interference. Different from the previous work, our proposed technique allocates same time slots to vehicles that are at least interference range apart. As a result, collisions due to hidden nodes are reduced. A major advantage of our proposed technique is that it does need any additional message or sensing time slot. Also, the proposed technique does not need to adapt the transmit power of vehicles. However, RSU needs to periodically evaluate the interference range within its coverage range. Moreover, vehicles calculate their allocated time slots using their GPS location and interference range parameter received from the RSU.

\section{Proposed Safety Message Transmission Technique}

In this section, we discuss the proposed safety message transmission technique for vehicular networks. We assume that vehicles are equipped with IEEE 802.11p enabled wireless transceivers. Moreover, each vehicle calculates its current location using the Global Position System (GPS) and shares it with other vehicles in regular safety messages.

\subsection{Interference Range Calculation Using Analytical Model}

To understand the hidden node problem in VANETs, let us consider Fig. 1. The figure shows a hidden node scenario where vehicle 2 receives a packet from vehicle 3 , at the same time it is busy receiving a packet from vehicle 1 . As a result, a collision can occur at vehicle 2 and it may not be able to receive any of the two packets. Such a scenario will be common in VANETs due to the broadcast nature of the CAM packets. Every vehicle will broadcast the cooperative awareness message within a CCHI (46 ms). Due to a short CCHI and broadcast communication used for safety messages, the hidden node problem will cause a large number of collisions.

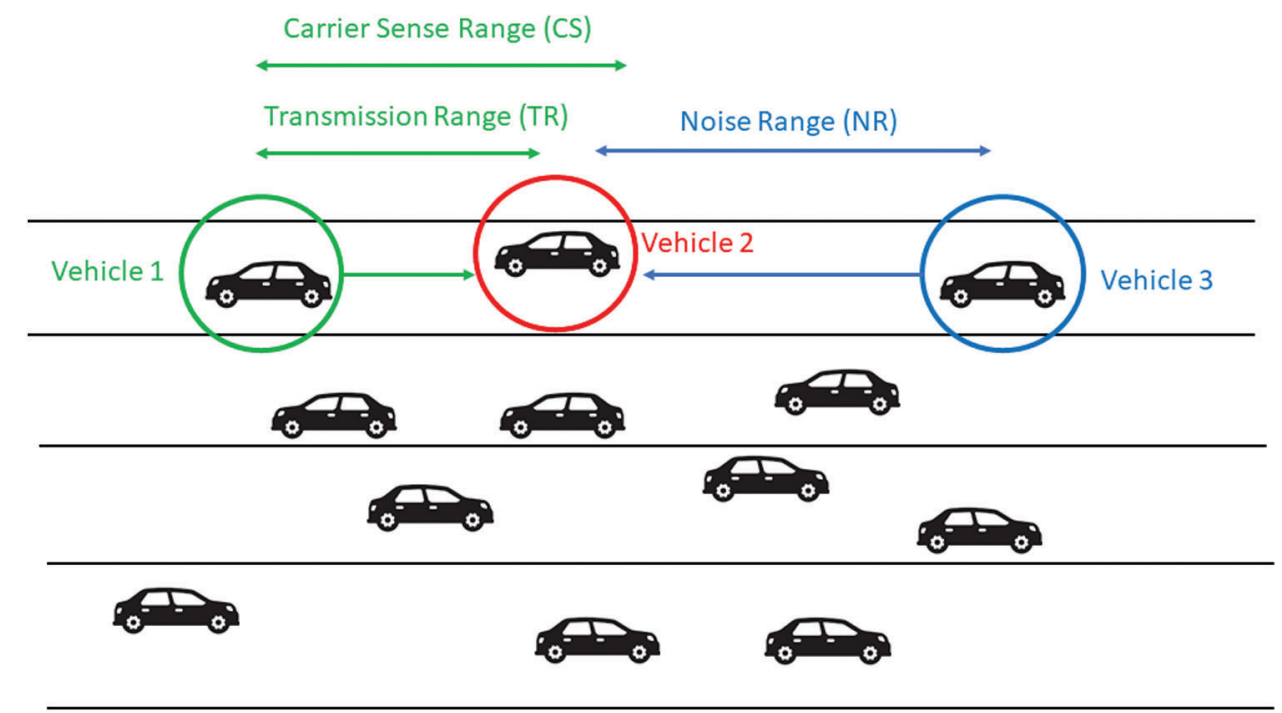

Figure 1: Simultaneous transmissions in the vehicular network (Here TR $=$ Transmission Range, $\mathrm{CS}=$ Carrier Sense Range, NR = Noise Range)

A wireless node has three different regions around it from a communication perspective namely transmission range (TR), carrier sense range (CSR), and noise range (NR). The transmission range of a vehicle is defined as the distance up to which a vehicle can successfully decode the received packet due 
to having a received power greater than the reception threshold $\left(\mathrm{Rx}_{\mathrm{Th}}\right)$. The carrier sense range of a vehicle is the distance up to which a vehicle can sense the packet as a result of having a received power greater than the carrier sense threshold $\left(\mathrm{CS}_{\mathrm{TH}}\right)$. Similarly, the noise range of a vehicle is the distance up to which the received power is greater than the noise threshold $\left(\mathrm{N}_{\mathrm{Th}}\right)$. All signals below the noise threshold are not detected by the wireless transceiver. If a vehicle receives two packets simultaneously, it can capture the stronger of the two packets if its power is greater than the power of the other packet by capture threshold $\left(\mathrm{Cp}_{\mathrm{Th}}\right)$. This effect is commonly known as the capture effect. The capture threshold value is normally equal to the required Signal to Interference plus Noise Threshold (SINR) for correct packet reception which is given by

$C p_{T H}=S I N R=R x_{T H}-N_{T H}(d B)=\frac{R x_{T H}}{N_{T H}}$

The TR, CSR, and NR are not deterministic in VANETs due to received power fluctuations as a result of fading. However, in this paper, the TR, CSR, and NR values are referred to as those obtained from a deterministic propagation model.

To gain more insight into the hidden problem, let us calculate the maximum interference range of a vehicle. From Fig. 3, we can see that the least power at which the packet from vehicle 1 is correctly decoded at vehicle 2 will be at a distance equal to the transmission range (TR). At this separation between vehicle 1 and vehicle 2, we will calculate the maximum distance of vehicle 3 which can cause a collision to the packet from vehicle 1 . This will give us the maximum interference range value. We can see from Fig. 3 that vehicle 1 can sense packets within its CSR. Only packets outside the CSR of vehicle 1 can cause a simultaneous transmission with vehicle 1 . On the other hand, vehicle 2 can receive interference from the vehicles within its NR. To successfully capture a packet from vehicle 1 in case of simultaneous transmission, the following condition must be satisfied according to capture effect.

$\frac{P_{1}}{P_{3}}>C p_{T H}$

where $P_{1}$ and $P_{3}$ are the received power values of vehicle 1 and vehicle 3 at vehicle 2 respectively.

The received power value at any distance $d$ is given by the following equation according to the deterministic propagation model [21]

$P_{r}(d)=P_{t} * P_{L}\left(d^{\gamma}\right)=\frac{P_{t} * C}{d^{\gamma}}$

where $\mathrm{P}_{\mathrm{t}}$ is the transmitted power, $\mathrm{P}_{\mathrm{L}}$ is the path loss which is a function of propagation distance and $\gamma$ is the path loss exponent. Path loss has an inverse relation with the propagation distance and can be written as the product of a constant factor (C) and inverse of the propagation distance as shown in Eq. (3). We assume that all vehicles have the same transmit power. Vehicle 1 is at a distance equal to TR from vehicle 2 . Let the distance between vehicle 3 and vehicle 2 be X. The ratio of P1 and P3 is given as follows.

$\frac{P 1}{P 3}=\left(\frac{P_{t} * C}{T R^{\gamma}}\right) *\left(\frac{X^{\gamma}}{P_{t} * C}\right)=\frac{X^{\gamma}}{T R^{\gamma}}$

Eq. (4) shows that the ratio of power at a receiver from two transmitter vehicles depends only on the propagation distances if the transmitted power is equal. As a result, the packet received at a receiver depends only on the propagation distances of the transmitting vehicles independent of the transmit power. 
By putting the received power ratio in Eq. (2) we get

$\frac{X^{\gamma}}{T R^{\gamma}}>C p_{T H}$

$X>C p_{T H^{\gamma}}{ }^{\frac{1}{\gamma}} * T R$

The transmission range can be evaluated using Eq. (3) by putting a received power value of $\mathrm{Rx}_{\mathrm{Th}}$.

$T R=\left(\frac{P_{t} * P_{L}}{R x_{T H}}\right)^{\frac{1}{\gamma}}$

By putting values of TR from Eq. (6) and Cp from Eq. (1) in Eq. (5), we get

$$
\begin{aligned}
& X>\left(\frac{R x_{T H}}{N_{T H}}\right)^{\frac{1}{\gamma}}\left(\frac{P_{t} * P_{L}}{R x_{T H}}\right)^{\frac{1}{\gamma}} \\
& X>\left(\frac{P_{t} * P_{L}}{N_{T H}}\right)^{\frac{1}{\gamma}}
\end{aligned}
$$

where

$N R=\left(\frac{P_{t} * P_{L}}{N_{T H}}\right)^{\frac{1}{\gamma}}$

The above result shows that vehicle 2 can correctly decode the packet from vehicle 1 only if vehicle 3 is located at a distance greater than the NR of vehicle 2 . Therefore, the maximum interference range in the worst case when the transmitter and receiver are separated by the transmission range will be equal to the noise range of the receiver. The potential hidden nodes for a vehicle exist between the carrier sense range of the transmitter and the noise range of the receiver as shown in Fig. 1. The above results can vary depending on the value of the capture threshold and can easily be evaluated using the above technique. The above result shows that a higher transmission range results in a higher noise range and a greater number of potential hidden node interferers.

\subsection{Safety Message Transmission Procedure}

Using the observations from the analytical model, we divide the road length of NR into small clusters of size $D_{c}$. The rationale behind using NR is that time slots can only be reused after this distance to avoid interference due to hidden vehicles. Each cluster is thus allocated a separate time slot of duration $\mathrm{T}_{\mathrm{c}}$ within CCHI. Vehicles use their current location to compute their current cluster number within NR, and hence only transmit at their allocated time slot.

\footnotetext{
Algorithm 1: Safety Message Transmission Procedure

Input: Noise Range (NR), Safety message transmission time Ts, Control Channel Interval Time $\left(\mathrm{T}_{\mathrm{CCHI}}\right)$

Calculate Number of clusters within $N R=N R / D_{c}$

Calculate Number of time slots within CCHI, $T_{\text {slots }}=T_{C C H I} / T_{S}$

Calculate $D_{c}=N R / \mathrm{T}_{\text {slots }}$

Each vehicle uses GPS location to find its cluster number within the NR

Each vehicle is allocated the time slot according to its cluster number within the NR
} 


\section{Performance Evaluation}

In this section, we present the simulation parameters and discuss the performance evaluation results. We used the NS-3 simulator to implement the proposed technique. The simulation parameters are discussed in Tab. 2. We consider 4 lanes in a highway scenario where vehicle density is taken as 50-200 vehicles $/ \mathrm{km}$. This corresponds to a congested scenario. Vehicle speeds are varied between 15 and $25 \mathrm{~m} / \mathrm{s}$. The transmission range for safety message transmissions is $300 \mathrm{~m}$. Transmit power of vehicles is taken as $23 \mathrm{dBm}$ and noise power is taken as $-114 \mathrm{dBm}$. We use two ray ground propagation model. We also use the Nakagami-m fading model in the simulations, where the $\mathrm{m}$ value is taken as $1-5$. Control channel duration is taken as $50 \mathrm{~ms}$. In IEEE $802.11 \mathrm{p}$, time is divided into control channel and service channel durations. Half of the time is dedicated to the control channel and the rest half of the time is reserved for service applications. Safety messages are transmitted over the control channel. The size of the safety message used is 300 bytes, and the packet generation rate is taken as 10 packets/second.

Table 2: Simulation parameters

\begin{tabular}{ll}
\hline Parameters & Values \\
\hline Vehicle Density & $50-200$ vehicles $/ \mathrm{km}$ \\
Vehicle speed & $15-25 \mathrm{~m} / \mathrm{s}$ \\
Number of Lanes & 4 \\
Transmission Range & $300 \mathrm{~m}$ \\
Data Rate & $6 \mathrm{Mbps}$ \\
Transmit Power & $23 \mathrm{dBm}$ \\
Noise Power & $-114 \mathrm{dBm}$ \\
Propagation Model & Two ray ground \\
Fading model & Nakagami-m $(\mathrm{m}=1,3,5)$ \\
Control Channel Duration $\mathrm{T}_{\mathrm{CCHI}}$ & 50 ms \\
Safety message size & 300 bytes \\
Safety message generation rate & 10 per second \\
\hline
\end{tabular}

We use two metrics to evaluate the performance of vehicular networks in this paper. The first is the packet reception ratio which is defined as the number of packets that are correctly received at receiving vehicles divided by the number of packets that are transmitted by transmitter vehicles. The second metric used is the ratio of packets that faced collisions. This is defined as the number of packets that are lost due to simultaneous transmissions at receiving vehicles divided by the number of packets that are received by the receiving vehicles. We compare the results of our proposed technique with the standard CSMA/CA protocol used in IEEE 802.11p.

In Fig. 2, we show the packet reception ratio at different fading levels i.e., Nakagami-m values and vehicle density of 200 vehicles $/ \mathrm{km}$. It can be seen that the packet reception ratio for the proposed technique remains above 0.95 for different $\mathrm{m}$ values. At higher $\mathrm{m}$ values, the packet reception ratio is high as there are no packet losses due to fading. At $m=1$, the packet reception ratio falls because of fading. In comparison, the CSMA/CA protocol achieves a packet reception ratio between 0.55 and 0.75 . The higher packet reception ratio by the proposed technique is due to the intelligent scheduling of transmissions to avoid collisions. 


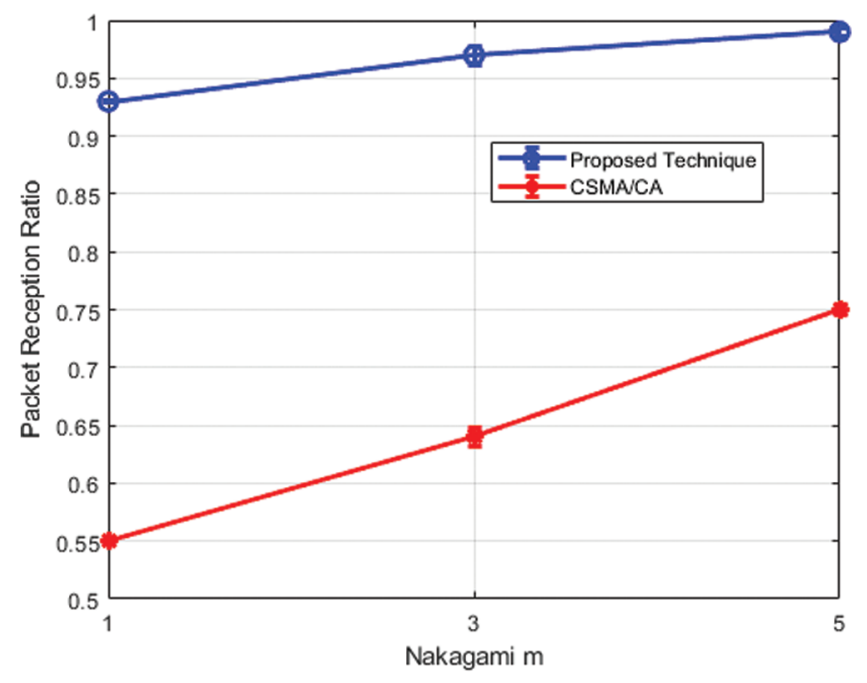

Figure 2: Packet Reception Ratio at different $\mathrm{m}$ values (Vehicle Density $=200$ vehicles $/ \mathrm{km}$ )

Fig. 3 shows the packet reception values of vehicles at different vehicle densities at $m=3$. Results show that the packet reception ratio is improved at lower vehicle densities as the number of simultaneous transmissions is low in number, and hence channel load on the network is reduced. While the proposed technique shows a packet reception ratio of higher than 0.97 , the CSMA/CA protocol has a packet reception ratio of around 0.64 at a vehicle density of 200 vehicles $/ \mathrm{km}$. As CSMA/CA protocol does not have any mechanism to overcome collisions due to simultaneous transmissions, it suffers from a low packet reception ratio. This can result in the loss of critical safety packets and compromise safety applications in vehicular networks.

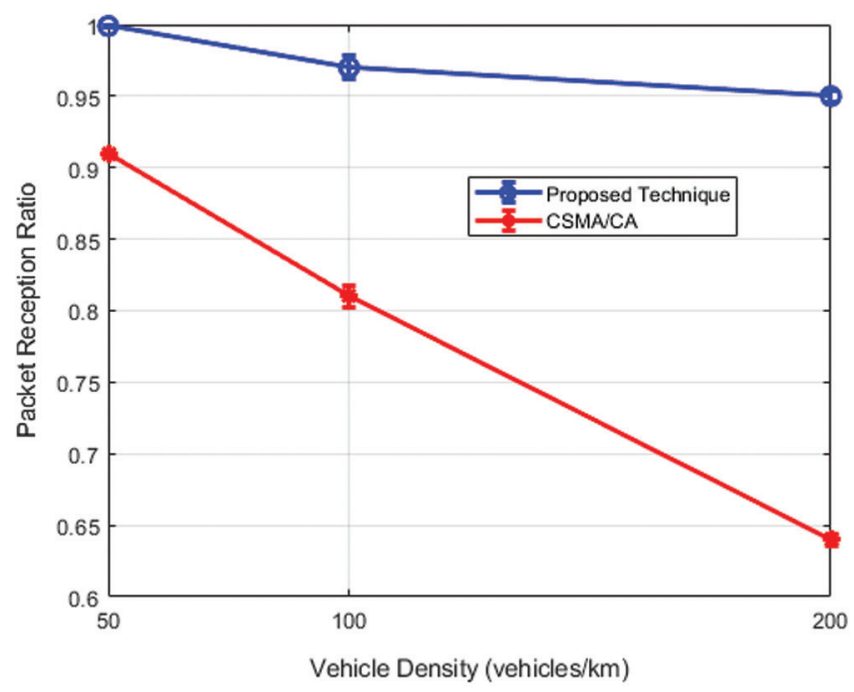

Figure 3: Packet Reception Ratio at vehicle densities $(\mathrm{m}=3)$

In Fig. 4, we show the ratio of packets that faced collisions at different $m$ values and vehicle density of 200 vehicles $/ \mathrm{km}$. The proposed technique reduces the ratio of collisions to less than 0.005 . On the other hand, $\mathrm{CSM} / \mathrm{CA}$ technique faces a collision ratio of up to 0.22 . This is caused by the large number of hidden nodes present in the vehicular network. 
IASC, 2022, vol.33, no.1

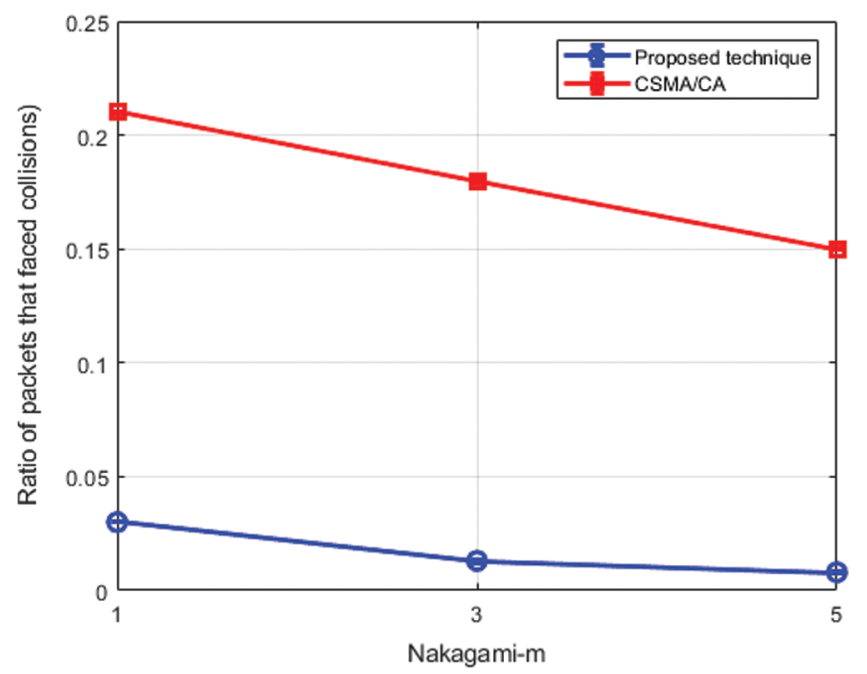

Figure 4: Ratio of packets that faced collisions at different $\mathrm{m}$ values (Vehicle Density $=200$ vehicles $/ \mathrm{km}$ )

The result in Fig. 5 presents the ratio of packets that faced collisions at different vehicle densities. The $\mathrm{m}$ value is taken as 3 . The collision ratio in CSMA/CA goes up to 0.18 whereas, in the proposed technique, it remains below 0.02. This significant advantage gained by the proposed technique in terms of reduced collisions is enabled by scheduling simultaneous transmissions at a distance greater than the noise range. Hence, the reliability of safety messages is improved significantly.

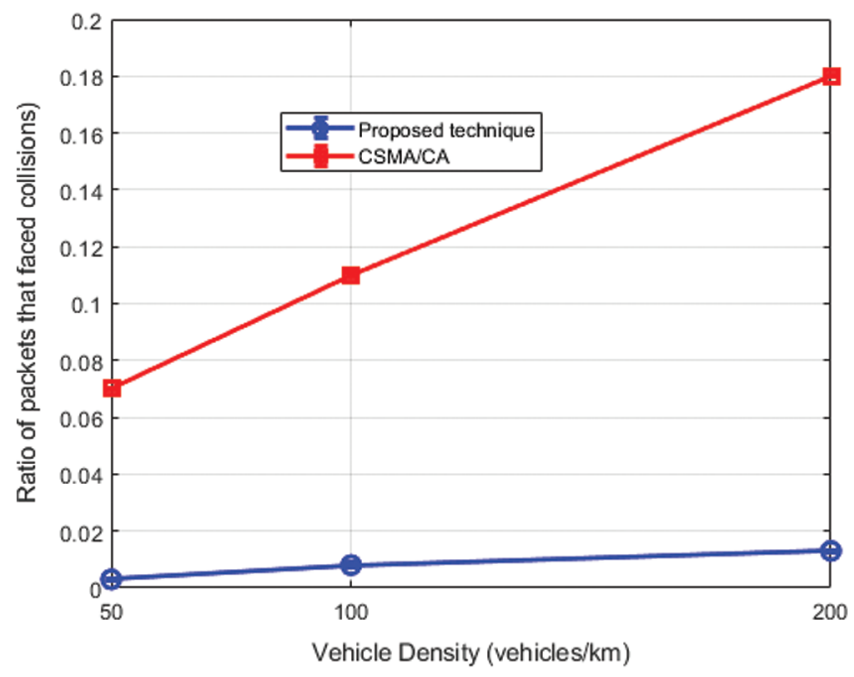

Figure 5: Ratio of packets that faced collisions at vehicle densities $(\mathrm{m}=3)$

From the above results, it can be noted that the proposed technique maintains a packet reception ratio of above $90 \%$ in different scenarios such as fading conditions and vehicle densities. This is achieved by scheduling simultaneous transmissions sufficient distance apart so that interference due to hidden nodes is mitigated. For the standard CSMA/CA technique, the packet reception ratio significantly falls at high vehicle densities and high fading intensity values. As safety applications require high packet reception ratio, the proposed technique is suited for the vehicular networks. 


\section{Conclusion}

Vehicular networks use regular sharing of safety messages to develop a map of neighborhood vehicles. As data sharing in vehicular networks is ad hoc, it can result in collisions due to hidden nodes. As a result, application reliability is compromised. To address this challenge, we propose an interference-aware transmission mechanism in this paper. We present an analytical model in this paper to find out the interference range of vehicles in a highway scenario. Furthermore, we schedule the safety message transmissions such that vehicles that can cause collisions with each other are allocated different time slots. We simulate the proposed technique in a realistic highway scenario in the NS-3 simulator. Results highlight the improvement achievement by the proposed algorithm in terms of packet reception ratio and reduced number of collisions as compared to the CSMA/CA-based IEEE 802.11p protocol.

Funding Statement: The authors extend their appreciation to the Deputyship for Research \& Innovation, Ministry of Education in Saudi Arabia for funding this research work the project number (442/84). Also, the authors would like to extend their appreciation to Taibah University for its supervision support.

Conflicts of Interest: The authors declare that they have no conflicts of interest to report regarding the present study.

\section{References}

[1] S. Bharati, W. Zhuang, L. V. Thanayankizil and F. Bai, "Link-layer cooperation based on distributed TDMA MAC for vehicular networks," IEEE Transactions on Vehicular Technology, vol. 66, no. 7, pp. 6415-6427, 2017.

[2] X. Zeng, M. Yu and D. Wang, "A new probabilistic multi-hop broadcast protocol for vehicular networks," IEEE Transactions on Vehicular Technology, vol. 67, no. 12, pp. 12165-12176, 2018.

[3] P. Wang, B. Di, H. Zhang, K. Bian and L. Song, "Cellular V2X communications in unlicensed spectrum: Harmonious coexistence with VANET in 5G systems," IEEE Transactions on Wireless Communications, vol. 17 , no. 8, pp. 5212-5224, 2018.

[4] X. He, J. Lv, J. Zhao, X. Hou and T. Luo, "Design and analysis of a short-term sensing-based resource selection scheme for C-V2X networks," IEEE Internet of Things Journal, vol. 7, no. 11, pp. 11209-11222, 2020.

[5] F. Abbas, P. Fan and Z. Khan, "A novel low-latency V2V resource allocation scheme based on cellular V2X communications," IEEE Transactions on Intelligent Transportation Systems, vol. 20, no. 6, pp. 2185-2197, 2019.

[6] B. Kang, J. Yang, J. Paek and S. Bahk, "Atomic: Adaptive transmission power and message interval control for C-V2X mode 4," IEEE Access, vol. 9, pp. 12309-12321, 2021.

[7] L. Yao, Y. Wang, X. Wang and G. Wu, "Cooperative caching in vehicular content centric network based on social attributes and mobility," IEEE Transactions on Mobile Computing, vol. 20, no. 2, pp. 391-402, 2021.

[8] M. Sepulcre and J. Gozalvez, "Heterogeneous V2V communications in multi-link and multi-RAT vehicular networks," IEEE Transactions on Mobile Computing, vol. 20, no. 1, pp. 162-173, 2021.

[9] K. Zhang, M. Peng and Y. Sun, "Delay-optimized resource allocation in fog-based vehicular networks," IEEE Internet of Things Journal, vol. 8, no. 3, pp. 1347-1357, 2021.

[10] U. Farooq, M. W. Shabir, M. A. Javed and M. Imran, "Intelligent energy prediction techniques for fog computing networks," Applied Soft Computing, vol. 111, no. 3, pp. 107682, 2021.

[11] M. Rahim, M. A. Javed, A. N. Alvi and M. Imran, "An efficient caching policy for content retrieval in autonomous connected vehicles," Transportation Research Part A: Policy and Practice, vol. 140, no. 3, pp. 142-152, 2020.

[12] F. Dewanta and M. Mambo, "BPT scheme: Establishing trusted vehicular fog computing service for rural area based on blockchain approach," IEEE Transactions on Vehicular Technology, vol. 70, no. 2, pp. 1752-1769, 2021.

[13] R. A. Nazib and S. Moh, "Reinforcement learning-based routing protocols for vehicular ad hoc networks: A comparative survey," IEEE Access, vol. 9, pp. 27552-27587, 2021. 
IASC, 2022, vol.33, no.1

[14] J. Garbiso, A. Diaconescu, M. Coupechoux and B. Leroy, "Fair self-adaptive clustering for hybrid cellularvehicular networks," IEEE Transactions on Intelligent Transportation Systems, vol. 22, no. 2, pp. 1225-1236, 2021.

[15] X. Wang and S. Cai, "Mobility management in vehicular named data networking," IEEE Sensors Letters, vol. 5, no. 8, pp. 1-4, 2021.

[16] U. M. Malik, M. A. Javed, S. Zeadally and S. U. Islam, "Energy efficient fog computing for $6 \mathrm{G}$ enabled massive IoT: Recent trends and future opportunities," IEEE Internet of Things Journal, pp. 1, 2021. https://ieeexplore.iee. org/document/9383089.

[17] Y. Cai, H. Zhang and Y. Fang, "A conditional privacy protection scheme based on ring signcryption for vehicular ad hoc networks," IEEE Internet of Things Journal, vol. 8, no. 1, pp. 647-656, 2021.

[18] M. A. Javed and S. Zeadally, "Ai-empowered content caching in vehicular edge computing: Opportunities and challenges," IEEE Network, vol. 35, no. 3, pp. 109-115, 2021.

[19] S. Zeadally, M. A. Javed and E. B. Hamida, "Vehicular communications for ITS: Standardization and challenges," IEEE Communications Standards Magazine, vol. 4, no. 1, pp. 11-17, 2020.

[20] S. Gyawali, S. Xu, Y. Qian and R. Q. Hu, "Challenges and solutions for cellular based V2X communications," IEEE Communications Surveys \&Tutorials, vol. 23, no. 1, pp. 222-255, 2021.

[21] C. Phillips, D. Sicker and D. Grunwald, "A survey of wireless path loss prediction and coverage mapping methods," IEEE Communications Surveys \& Tutorials, vol. 15, no. 1, pp. 255-270, 2013. 